\title{
The discoveries of WASP-91b, WASP-105b and WASP-107b: Two warm Jupiters and a planet in the transition region between ice giants and gas giants ${ }^{\star, \star \star}$
}

D. R. Anderson ${ }^{1}$, A. Collier Cameron ${ }^{2}$, L. Delrez ${ }^{3,4}$, A. P. Doyle ${ }^{5}$, M. Gillon ${ }^{3}$, C. Hellier ${ }^{1}$, E. Jehin ${ }^{3}$, M. Lendl ${ }^{3,6}$, P. F. L. Maxted ${ }^{1}$, N. Madhusudhan ${ }^{4}$, F. Pepe ${ }^{6}$, D. Pollacco ${ }^{5}$, D. Queloz ${ }^{4}$, D. Ségransan ${ }^{6}$, B. Smalley ${ }^{1}$, A. M. S. Smith ${ }^{1,7,8}$, A. H. M. J. Triaud ${ }^{6,9,10,11}$, O. D. Turner ${ }^{1}$, S. Udry ${ }^{6}$, and R. G. West ${ }^{5}$

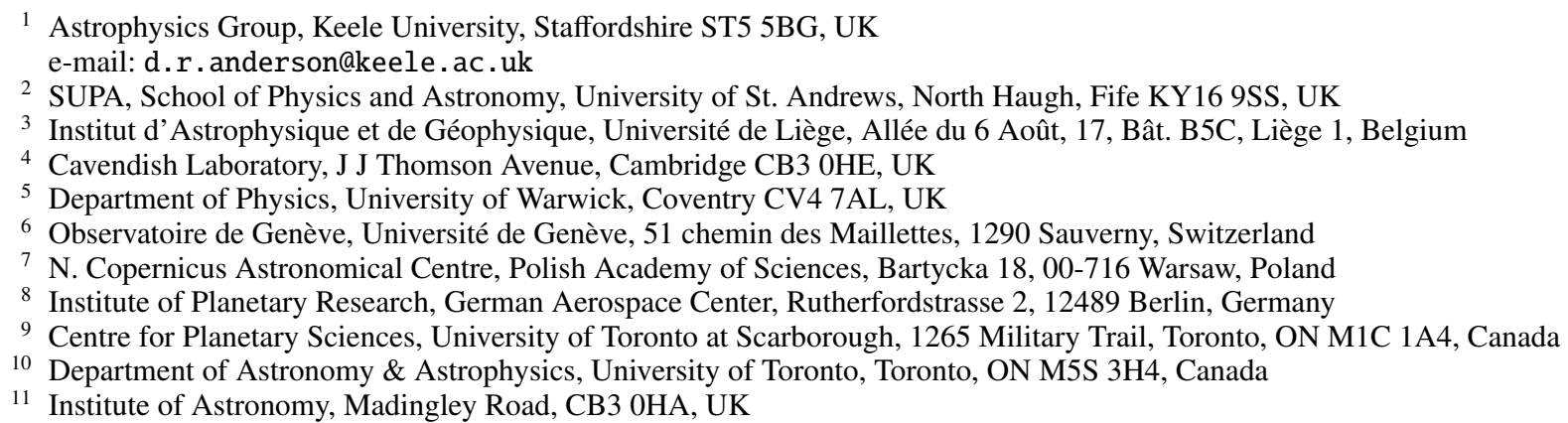

Received 14 January 2017 / Accepted 11 February 2017

\begin{abstract}
We report the discoveries of three transiting exoplanets. WASP-91b is a warm Jupiter $\left(1.34 M_{\mathrm{Jup}}, 1.03 R_{\mathrm{Jup}}\right)$ in a 2.8 -day orbit around a metal-rich K3 star. WASP-105b is a warm Jupiter (1.8 $\left.M_{\text {Jup }}, 0.96 R_{\text {Jup }}\right)$ in a 7.9-day orbit around a metal-rich K2 star. WASP-107b is a warm super-Neptune/sub-Saturn $\left(0.12 M_{\mathrm{Jup}}, 0.94 R_{\mathrm{Jup}}\right)$ in a 5.7-day orbit around a solar-metallicity K6 star. Considering that giant planets seem to be more common around stars of higher metallicity and stars of higher mass, it is notable that the hosts are all metalrich, late-type stars. With orbital separations that place both WASP-105b and WASP-107b in the weak-tide regime, measurements of the alignment between the planets' orbital axes and their stars' spin axes may help us to understand the inward migration of shortperiod, giant planets. The mass of WASP-107b $\left(2.2 M_{\mathrm{Nep}}, 0.40 M_{\mathrm{Sat}}\right)$ places it in the transition region between the ice giants and gas giants of the Solar System. Its radius of $0.94 R_{\text {Jup }}$ suggests that it is a low-mass gas giant with a $\mathrm{H} / \mathrm{He}$-dominated composition. The planet thus sets a lower limit of $2.2 M_{\mathrm{Nep}}$ on the planetary mass above which large gaseous envelopes can be accreted and retained by proto-planets on their way to becoming gas giants. We may discover whether WASP-107b more closely resembles an ice giant or a gas giant by measuring its atmospheric metallicity via transmission spectroscopy, for which WASP-107b is a very good target.
\end{abstract}

Key words. planets and satellites: formation - planets and satellites: individual: WASP-91b - planetary systems planets and satellites: individual: WASP-105b - planets and satellites: individual: WASP-107b

\section{Introduction}

The observation that the fraction of stars with giant planets increases with both stellar metallicity and mass is suggestive of planetary formation by core accretion (e.g. Santos et al. 2004; Johnson et al. 2010). Under the core accretion model (e.g. Pollack et al. 1996), a gas giant results when planetesimals coagulate to form a rocky core, which then accretes a gaseous envelope. The Solar System's gas giants, Jupiter and Saturn (0.30 $\left.M_{\text {Jup }}, 0.84 R_{\text {Jup }}\right)$, are more than $90 \% \mathrm{H} / \mathrm{He}$ by mass,

\footnotetext{
* Based on observations made with: the WASP-South photometric survey instrument, the 0.6-m TRAPPIST robotic imager, and the EulerCam camera and the CORALIE spectrograph mounted on the $1.2-\mathrm{m}$ Euler-Swiss telescope.

$\star \star$ The photometric time-series and radial-velocity data used in this work are only available at the CDS via anonymous ftp to cdsarc.u-strasbg.fr (130.79.128.5) or via http://cdsarc.u-strasbg.fr/viz-bin/qcat?]/A+A/604/A110
}

which contrasts with the figure of $20 \%$ for the less massive $\left(\sim 0.05 M_{\text {Jup }}\right)$ and smaller $\left(\sim 0.35 R_{\text {Jup }}\right)$ ice giants, Neptune and Uranus (Guillot 2005). One challenge faced by models attempting to explain the formation of Neptune and Uranus is to avoid the runaway gas accretion that otherwise would have turned the planets into gas giants (e.g. Helled \& Bodenheimer 2014).

Giant planets in few-day orbits, or "warm/hot Jupiters", are thought to have formed farther out and then migrated inwards via interaction with the gas disc or via a high-eccentricity pathway (Lin et al. 1996; Rasio \& Ford 1996). Planet-disc migration is expected to preserve alignment between the stellar spin and planetary orbital axes (e.g. Marzari \& Nelson 2009), whereas high-eccentricity migration is expected to produce a broad range of misalignments (e.g. Fabrycky \& Tremaine 2007). The ensemble of available measurements has been interpreted as evidence that hot Jupiters arise via high-eccentricity migration (Winn et al. 2010), though planet-disc migration is likely to play a role (e.g. Anderson et al. 2015b). 
Table 1. Summary of observations.

\begin{tabular}{|c|c|c|c|c|}
\hline Facility & Date & $N_{\text {obs }}$ & $T_{\exp }[\mathrm{s}]$ & Filter \\
\hline \multicolumn{5}{|l|}{ WASP-91: } \\
\hline WASP-South & 2010 Jun.-2011 Dec. & 16800 & 30 & $\operatorname{Broad}(400-700 \mathrm{~nm})$ \\
\hline Euler/CORALIE & 2012 Jan.-Sep. & 12 & 1800 & Spectroscopy \\
\hline TRAPPIST & 2012 Jul. 20 & 578 & 15 & $I+z^{\prime}$ \\
\hline TRAPPIST & 2012 Aug. 31 & 934 & 12 & $I+z^{\prime}$ \\
\hline Euler/EulerCam & 2012 Oct. 12 & 172 & 70 & Gunn- $r$ \\
\hline TRAPPIST & 2012 Oct. 29 & 395 & 15 & $R$ \\
\hline Euler/EulerCam & 2013 Jun. 04 & 131 & 110 & Gunn- $r$ \\
\hline TRAPPIST & 2013 Jun. 04 & 794 & 8 & $I+z^{\prime}$ \\
\hline \multicolumn{5}{|l|}{ WASP-105: } \\
\hline WASP-South & 2010 Jun.-2011 Dec. & 11200 & 30 & Broad $(400-700 \mathrm{~nm})$ \\
\hline Euler/CORALIE & 2013 Aug.-2014 Jan. & 25 & 1800 & Spectroscopy \\
\hline TRAPPIST & 2013 Jul. 23 & 703 & 10 & $I+z^{\prime}$ \\
\hline Euler/EulerCam & 2013 Sep. 24 & 288 & 70 & Gunn- $r$ \\
\hline TRAPPIST & 2013 Sep. 24 & 906 & 12 & $I+z^{\prime}$ \\
\hline Euler/EulerCam & 2013 Oct. 02 & 257 & 70 & Gunn- $r$ \\
\hline TRAPPIST & 2013 Nov. 26 & 792 & 12 & $I+z^{\prime}$ \\
\hline TRAPPIST & 2014 Dec. 09 & 1072 & 7 & $I+z^{\prime}$ \\
\hline \multicolumn{5}{|l|}{ WASP-107: } \\
\hline WASP-South & 2009 Feb.-2010 Jun. & 9350 & 30 & $\operatorname{Broad}(400-700 \mathrm{~nm})$ \\
\hline Euler/CORALIE & 2011 Mar-2014 Jan. & 32 & 1800 & Spectroscopy \\
\hline TRAPPIST & 2013 Jan. 09 & 532 & 10 & $z^{\prime}$ \\
\hline Euler/EulerCam & 2013 Feb. 18 & 264 & 50 & Gunn- $r$ \\
\hline TRAPPIST & 2013 Feb. 18 & 864 & 10 & $z^{\prime}$ \\
\hline TRAPPIST & 2013 May 15 & 814 & 10 & $z^{\prime}$ \\
\hline Euler/EulerCam & 2014 Feb. 02 & 154 & 75 & Gunn- $r$ \\
\hline TRAPPIST & 2014 Apr. 06 & 692 & 10 & $z^{\prime}$ \\
\hline
\end{tabular}

In this paper, we present the discoveries of three transiting exoplanets by the WASP survey: WASP-91b and WASP$105 \mathrm{~b}$ are warm Jupiters orbiting metal-rich, early/mid-K stars; and WASP-107b is a warm super-Neptune/sub-Saturn orbiting a solar-metallicity, late-K star.

\section{Observations}

WASP-South images one third of the visible South-African sky (avoiding the galactic plane and the south pole) every $\sim 10 \mathrm{~min}$ and is sensitive to the detection of giant planets transiting bright stars $(V=9-13)$. The survey and the search techniques are described in Pollacco et al. (2006) and Collier Cameron et al. (2006, 2007).

We routinely investigate the promising transit signals that we find in WASP lightcurves with the EulerCam imager and the CORALIE spectrograph, both of which are mounted on the 1.2-m Euler-Swiss telescope, and the 0.6-m TRAPPIST imager and (Lendl et al. 2012; Queloz et al. 2000; Gillon et al. 2011; Jehin et al. 2011). We provide a summary of our observations of the three target stars in Table 1. TRAPPIST performed meridian flips at the following times (BJD-2 450000 ): 6560.756 (WASP105 transit of 2013 September 24); 7001.546 (WASP-105 transit of 2014 December 9); 6428.575 (WASP-107 transit of 2013 May 15); and 6754.683 (WASP-107 transit of 2014 April 6). We partioned the resulting lightcurves prior to fitting to allow for flux offsets. We interpret the bump in the lightcurve of WASP107 around mid-transit on 2013 Feb. 18 as having been caused by the planet occulting a star spot.

The radial-velocity (RV) measurements that we computed from the CORALIE spectra exhibit variations with similar periods as the photometric dimmings seen in the WASP lightcurves and with amplitudes consistent with planetary-mass companions. The photometry and RVs are plotted for each system in Figs. 1-3. The absence of a significant correlation between bisector span and RV supports our conclusion that the observed periodic dimmings and RV variations are caused by transiting planets (Fig. 4).
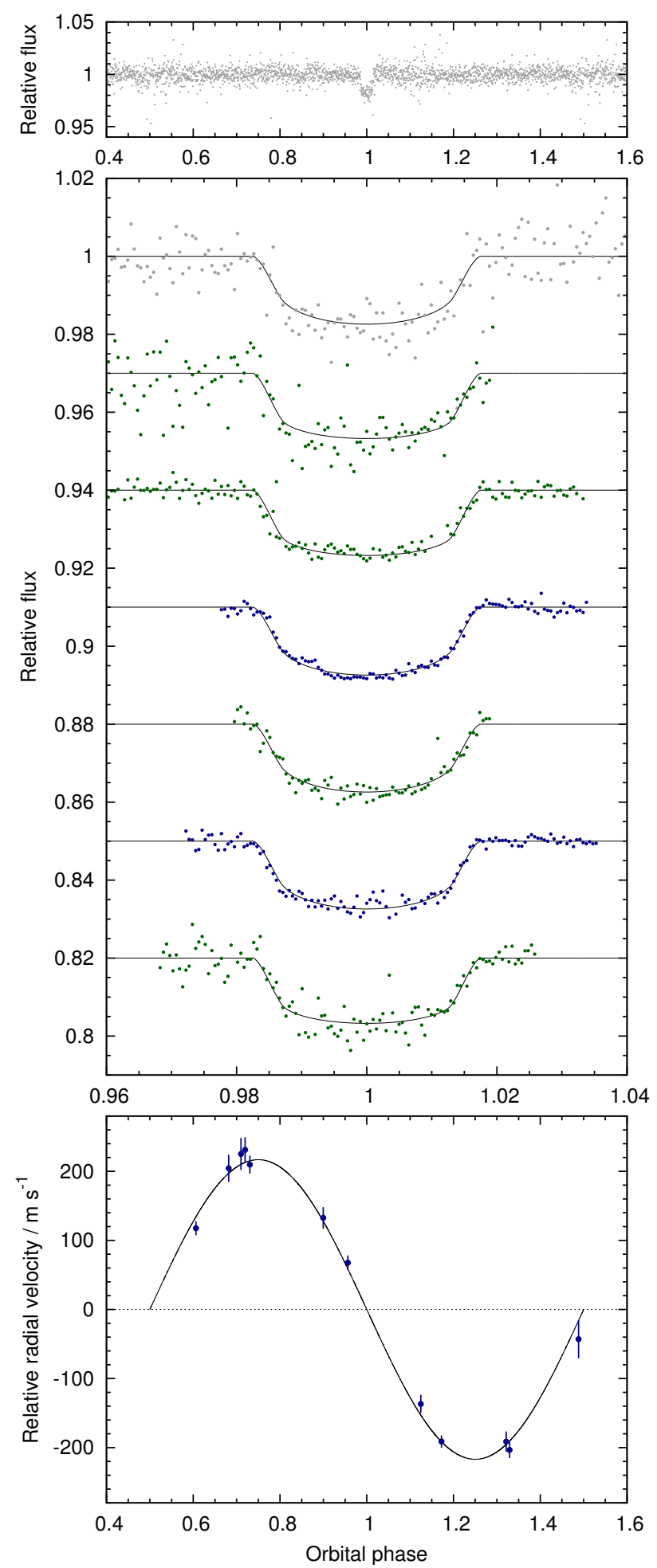

Fig. 1. WASP-91b discovery data. Top panel: WASP lightcurve folded on the transit ephemeris. Middle panel: transit lightcurves from WASP (grey), TRAPPIST (green) and EulerCam (blue), offset for clarity, binned with a bin width of two minutes, and plotted chronologically with the most recent at the bottom. The best-fitting transit model is superimposed. Bottom panel: the CORALIE radial velocities with the best-fitting circular orbital model.

\section{Stellar parameters from spectra}

The individual CORALIE spectra were co-added after correcting for the orbital motion of the star, giving average signal-to-noise 

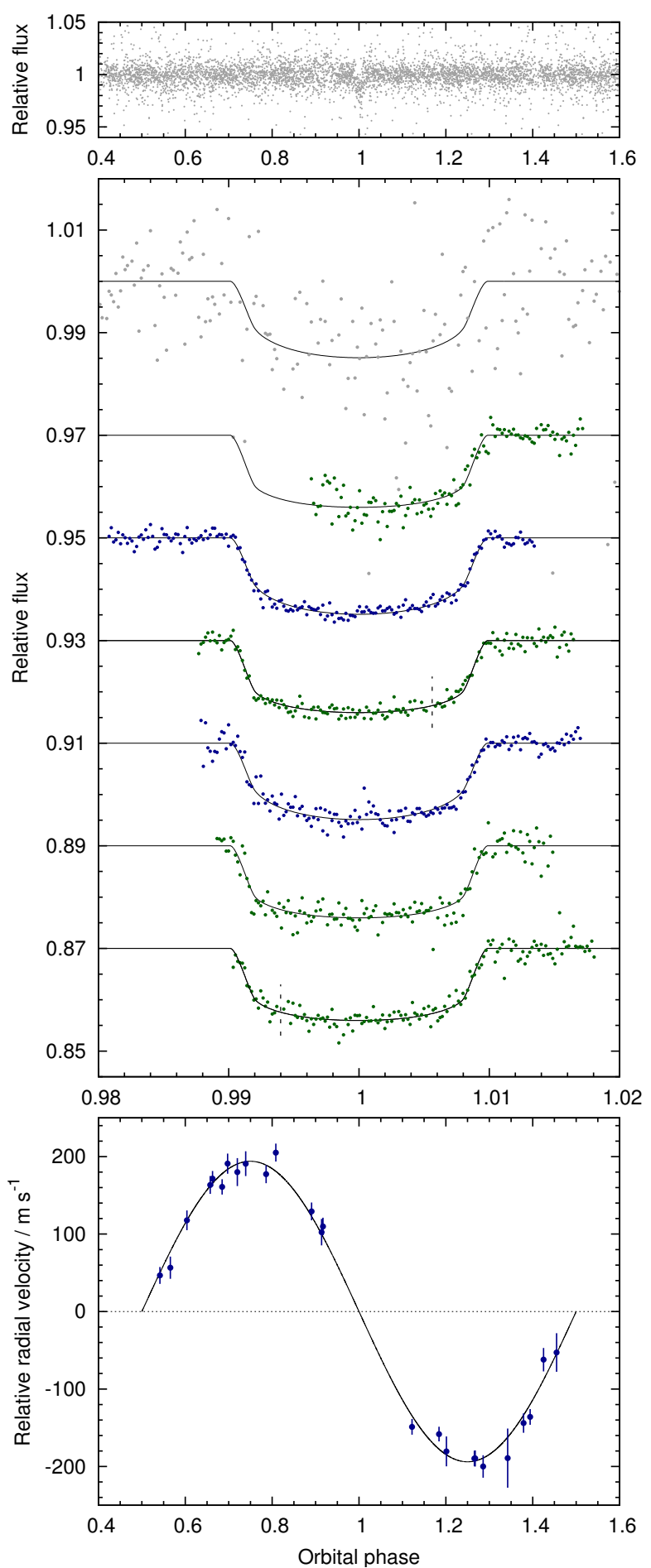

Fig. 2. WASP-105b discovery data. Caption as for Fig. 1. Data partioning due to TRAPPIST's meridian flips are indicated by vertical dashed lines.

ratios of 55:1, 80:1 and 120:1 for WASP-91, WASP-105 and WASP-107, respectively. We performed the spectral analysis using the procedures detailed in Doyle et al. (2013). For each star the effective temperature $\left(T_{\text {eff }}\right)$ was obtained using the $\mathrm{H} \alpha$ line and surface gravity $\left(\log g_{*}\right)$ was determined from the $\mathrm{Na} \mathrm{D}$ and $\mathrm{Mg} \mathrm{b}$ lines. Iron abundances were obtained from the analysis of equivalent width measurements of several unblended Fe I lines.

Projected equatorial rotation velocities $\left(v_{*} \sin I_{*}\right)$ were determined by fitting the profiles of the Fe I lines after convolving

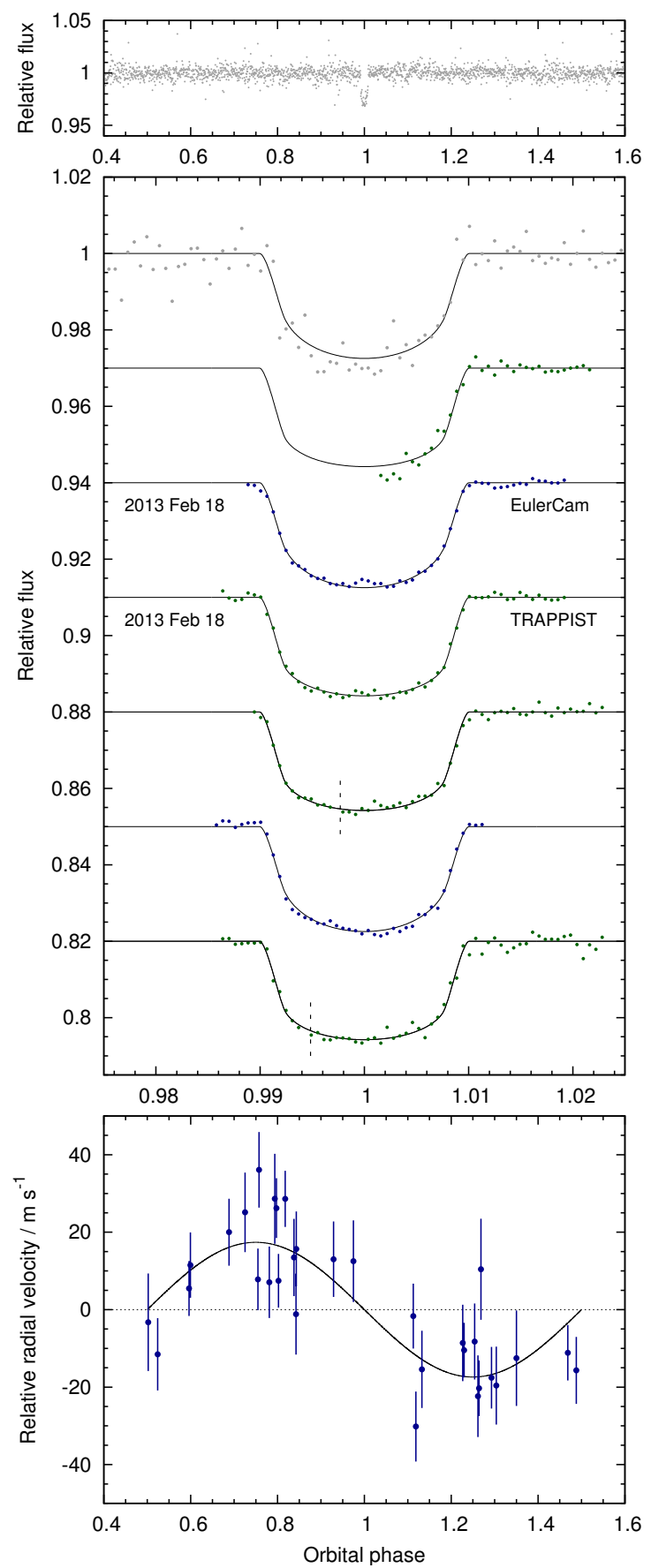

Fig. 3. WASP-107b discovery data. Caption as for Fig. 1. The planet appears to have passed over a star spot during the transit of 2013 Feb. 18, with a bump more evident in the lightcurve from EulerCam (Gunn $r$ ) than TRAPPIST (Sloan $z$ ). The difference is expected due to the bluer passband employed by EulerCam, in which the contrast between star spots and the surrounding photosphere will be greater. Also, the diameter of the TRAPPIST telescope is half that of the Euler telescope $(0.6 \mathrm{~m}$ versus $1.2 \mathrm{~m}$ ), so the data are noisier. Data partioning due to TRAPPIST's meridian flips are indicated by vertical dashed lines.

with the instrumental resolution $(R=55000)$. For WASP-105 a macroturbulent velocity of $0.9 \pm 0.3 \mathrm{~km} \mathrm{~s}^{-1}$ was adopted from (Gray 2008). In the cases of WASP-91 and WASP-107, macroturbulence was assumed to be zero, since for mid/late K stars it is expected to be lower than that of thermal broadening (Gray 2008). The results are given in the top panel of Table 2. 

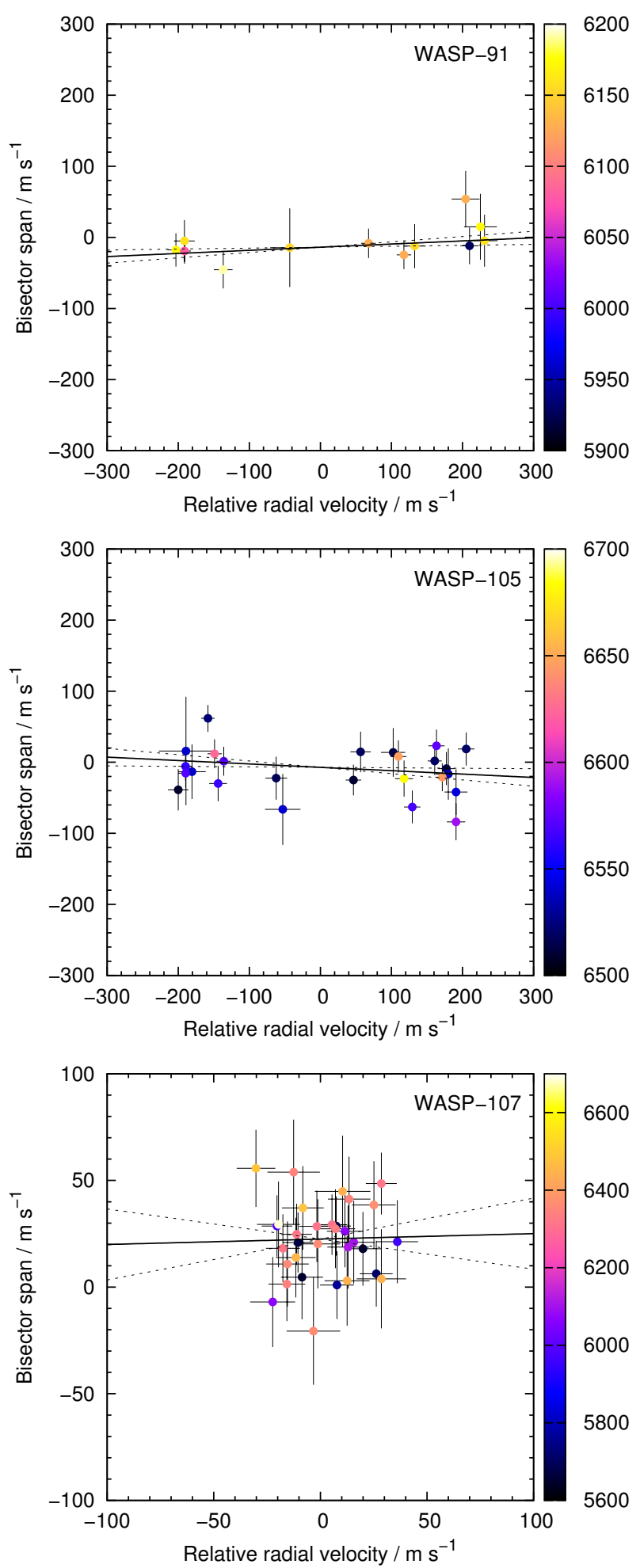

Fig. 4. Absence of correlation between bisector span and radial velocity for the three stars excludes transit mimics. The solid line is the best linear fit to the data and the dotted lines are the $1 \sigma$ limits on the gradient. The Julian date of the observation (BJD - 2450 000) is represented by the symbol colour.

\section{Stellar rotation from lightcurve modulation}

The WASP lightcurves of WASP-107 show a periodic modulation with an amplitude of about 0.4 per cent and a period of about 17 days. We assume this is due to the combination of the star's rotation and magnetic activity, i.e., star spots. We used the sinewave fitting method described in Maxted et al. (2011) to refine this estimate of the amplitude and period of the modulation. Variability due to star spots is not expected to be coherent on long timescales as a consequence of the finite lifetime of star-spots and differential rotation in the photosphere so we analysed the two seasons of data for WASP-107 separately. We removed the transit signal from the data prior to calculating the periodograms by subtracting a simple transit model from the lightcurve and also removed low-frequency noise by subtracting a straight line fit by least-squares to the data from each season. We calculated periodograms over 8192 uniformly spaced frequencies from 0 to 1.5 cycles/day. The false alarm probability (FAP) is calculated using a boot-strap Monte Carlo method also described in Maxted et al. (2011). The results are given in Table 3 and the periodograms and lightcurves are shown in Fig. 5. There is a clear signal at a period of $P=17.1 \mathrm{~d}$ in the 2010 season of data that is also in the data from 2009 at $P=17.3 \mathrm{~d}$, though at a lower significance. The periodogram of the data from 2009 also shows a peak at $P=8.3 \mathrm{~d}$, which we assume is the second-harmomic of the rotation period due of multiple spot groups on surface of the star during this observing season. The strongest peak in this periodogram at $1.134 \mathrm{~d}$ can then be ascribed to the 1-day alias of this second-harmomic.

Assuming this to be the case, we obtain a value for the rotation period of $P_{\text {rot }}=17 \pm 1 \mathrm{~d}$, where the error in this value is taken from the full-width at half-maximum of the peak in the periodogram of the data from 2010. This rotation period together with our estimate of the stellar radius (Sect. 5) implies a value for the stellar rotation velocity of $v_{*}=2.0 \pm 0.1 \mathrm{~km} \mathrm{~s}^{-1}$. This compares well with the spectroscopic estimate of the projected equatorial rotation velocity of $v_{*} \sin I_{*}=2.5 \pm 0.8 \mathrm{~km} \mathrm{~s}^{-1}$. We used a least-squares fit of a sinusoidal function and its first harmonic to model the rotational modulation in the lightcurves for each camera and season with the rotation period fixed at $P_{\text {rot }}=17.1 \mathrm{~d}$. We then subtracted this harmonic series fit from the original lightcurve prior to our analysis of the transit (Sect. 5).

For WASP-91 and WASP-105 a similar analysis lead to upper limits with 95 per cent confidence of $0.8 \mathrm{mmag}$ and $0.7 \mathrm{mmag}$ for the amplitude of any sinusoidal signal over the same frequency range.

\section{System parameters from the RV and transit data}

We determined the parameters of each system by fitting the photometric and radial-velocity data simultaneously using the current version of the Markov-chain Monte Carlo (MCMC) code described by Collier Cameron et al. (2007) and Anderson et al. (2015a). The transit lightcurves were modelled using the formulation of Mandel \& Agol (2002) and limb-darkening was accounted for using the four-parameter non-linear law of Claret (2000, 2004).

Stellar density is measured from the transit lightcurves, but we require a constraint on stellar mass for a full characterisation of the system. For that we used the BAGEMASs stellar evolution MCMC code of Maxted et al. (2015), using the values of $\rho_{*}$ from an initial MCMC run and the values of $T_{\text {eff }}$ and $[\mathrm{Fe} / \mathrm{H}]$ from the spectral analysis. From BAGEMASS we obtained values of stellar mass $\left(M_{*}\right)$ of $0.840 \pm 0.032 M_{\odot}, 0.891 \pm 0.047 M_{\odot}$ and $0.691 \pm 0.025 M_{\odot}$ for WASP-91, WASP-105 and WASP-107, respectively. In our final MCMC analyses, we drew a value of $M_{*}$ at each MCMC step from a normal distribution with mean and standard deviation equal to the BAGEMASS-derived values, but with an error bar larger by a factor 2 to allow for uncertainties 
Table 2. System parameters.

\begin{tabular}{|c|c|c|c|c|}
\hline Parameter & Symbol (Unit) & WASP-91 & WASP-105 & WASP-107 \\
\hline \multicolumn{5}{|c|}{ Stellar parameters, including from the spectra: } \\
\hline $\begin{array}{l}\text { Constellation } \\
\end{array}$ & & Tucana & Phoenix & Virgo \\
\hline Right Ascension & & $23^{\mathrm{h}} 51^{\mathrm{m}} 22 \mathrm{~s} 89$ & $01^{\mathrm{h}} 36^{\mathrm{m}} 40.24$ & $12^{\mathrm{h}} 33^{\mathrm{m}} 32^{\mathrm{s}} .84$ \\
\hline Declination & & $-70^{\circ} 09^{\prime} 10^{s} 2$ & $-50^{\circ} 39^{\prime} 32.5$ & $-10^{\circ} 08^{\prime} 46 \mathrm{~s} 1$ \\
\hline$V_{\mathrm{mag}}$ & & 12.0 & 12.1 & 11.6 \\
\hline$K_{\mathrm{mag}}$ & & 9.7 & 9.9 & 8.6 \\
\hline Spectral type ${ }^{a}$ & & K3 & $\mathrm{K} 2$ & K6 \\
\hline Stellar effective temperature & $T_{\text {eff }}(\mathrm{K})$ & $4920 \pm 80$ & $5070 \pm 130$ & $4430 \pm 120$ \\
\hline Stellar surface gravity & $\log g_{*}(\mathrm{cgs})$ & $4.3 \pm 0.2$ & $4.2 \pm 0.2$ & $4.5 \pm 0.1$ \\
\hline Projected equatorial rotation velocity & $v_{*} \sin I_{*} / \mathrm{km} \mathrm{s}^{-1}$ & $2.4 \pm 0.4$ & $1.7 \pm 1.9$ & $2.5 \pm 0.8$ \\
\hline Stellar metallicity ${ }^{b}$ & {$[\mathrm{Fe} / \mathrm{H}]$} & $+0.19 \pm 0.13$ & $+0.28 \pm 0.16$ & $+0.02 \pm 0.10$ \\
\hline Lithium abundance & $\log A(\mathrm{Li})$ & $<0.5$ & $<0.2$ & $<-0.3$ \\
\hline \multicolumn{5}{|l|}{ MCMC proposal parameters: } \\
\hline Orbital period & $P(\mathrm{~d})$ & $2.798581 \pm 0.000003$ & $7.87288 \pm 0.00001$ & $5.721490 \pm 0.000002$ \\
\hline Epoch of mid-transit & $T_{c}(\mathrm{BJD})$ & $2456297.7190 \pm 0.0002$ & $2456600.0765 \pm 0.0002$ & $2456514.4106 \pm 0.0001$ \\
\hline Transit duration & $T_{14}(\mathrm{~d})$ & $0.0976 \pm 0.0008$ & $0.1550 \pm 0.0006$ & $0.1147 \pm 0.0003$ \\
\hline Planet-to-star area ratio & $R_{\mathrm{p}}^{2} / \mathrm{R}_{*}^{2}$ & $0.0150 \pm 0.0003$ & $0.0120 \pm 0.0001$ & $0.0217 \pm 0.0002$ \\
\hline Impact parameter ${ }^{c}$ & $b$ & $0.51 \pm 0.04$ & $0.10 \pm 0.08$ & $0.09 \pm 0.07$ \\
\hline Stellar reflex velocity semi-amplitude & $K_{1}\left(\mathrm{~m} \mathrm{~s}^{-1}\right)$ & $217 \pm 5$ & $194 \pm 3$ & $17 \pm 2$ \\
\hline Systemic velocity & $\gamma\left(\mathrm{m} \mathrm{s}^{-1}\right)$ & $2782 \pm 4$ & $24676 \pm 2$ & $14160 \pm 2$ \\
\hline Orbital eccentricity & $e$ & 0 (adopted; $<0.07$ at $2 \sigma)$ & 0 (adopted; $<0.04$ at $2 \sigma)$ & 0 (adopted; $<0.4$ at $2 \sigma$ ) \\
\hline \multicolumn{5}{|l|}{ MCMC derived parameters: } \\
\hline Scaled orbital separation & $a / R_{*}$ & $9.1 \pm 0.3$ & $17.9 \pm 0.2$ & $18.2 \pm 0.1$ \\
\hline Orbital inclination & $i\left({ }^{\circ}\right)$ & $86.8 \pm 0.4$ & $89.7 \pm 0.2$ & $89.7 \pm 0.2$ \\
\hline Transit ingress/egress duration & $T_{12}=T_{34}(\mathrm{~d})$ & $0.0139 \pm 0.0009$ & $0.0154 \pm 0.0003$ & $0.0148 \pm 0.0002$ \\
\hline Stellar mass & $M_{*}\left(M_{\odot}\right)$ & $0.84 \pm 0.07$ & $0.89 \pm 0.09$ & $0.69 \pm 0.05$ \\
\hline Stellar radius & $R_{*}\left(R_{\odot}\right)$ & $0.86 \pm 0.03$ & $0.90 \pm 0.03$ & $0.66 \pm 0.02$ \\
\hline Stellar surface gravity & $\log g_{*}(\mathrm{cgs})$ & $4.49 \pm 0.03$ & $4.48 \pm 0.02$ & $4.64 \pm 0.01$ \\
\hline Stellar density & $\rho_{*}\left(\rho_{\odot}\right)$ & $1.3 \pm 0.1$ & $1.23 \pm 0.03$ & $2.45 \pm 0.05$ \\
\hline Planetary mass & $M_{\mathrm{P}}\left(M_{\mathrm{Jup}}\right)$ & $1.34 \pm 0.08$ & $1.8 \pm 0.1$ & $0.12 \pm 0.01$ \\
\hline Planetary radius & $R_{\mathrm{P}}\left(R_{\text {Jup }}\right)$ & $1.03 \pm 0.04$ & $0.96 \pm 0.03$ & $0.94 \pm 0.02$ \\
\hline Planetary surface gravity & $\log g_{\mathrm{P}}(\mathrm{cgs})$ & $3.46 \pm 0.03$ & $3.64 \pm 0.01$ & $2.49 \pm 0.05$ \\
\hline Planetary density & $\rho_{\mathrm{P}}\left(\rho_{\mathrm{J}}\right)$ & $1.2 \pm 0.1$ & $2.0 \pm 0.1$ & $0.14 \pm 0.02$ \\
\hline Orbital major semi-axis & $a(\mathrm{au})$ & $0.037 \pm 0.001$ & $0.075 \pm 0.003$ & $0.055 \pm 0.001$ \\
\hline Planetary equil. temperature ${ }^{d}$ & $T_{\text {eql }}(\mathrm{K})$ & $1160 \pm 30$ & $900 \pm 20$ & $770 \pm 60$ \\
\hline
\end{tabular}

Notes. ${ }^{(a)}$ Iron abundances are relative to the solar values of Asplund et al. (2009). ${ }^{(b)}$ Spectral type estimated from $T_{\text {eff }}$ using the table in Gray (2008). ${ }^{(c)}$ Impact parameter is the distance between the centre of the stellar disc and the transit chord: $b=a \cos i / R_{*} .{ }^{(d)}$ Equilibrium temperature calculated assuming zero albedo and efficient redistribution of heat from the planet's presumed permanent day-side to its night-side.

due to the unknown helium abundances and the effects of magnetic activity on the mass-radius relation.

In initial MCMC runs we modelled eccentric orbits, but for no system do we find compelling evidence of a non-circular orbit. We thus adopt circular orbits, which Anderson et al. (2012) argue is the prudent choice for short-period, Jupiter-mass planets in the absence of evidence to the contrary. We place $2 \sigma$ upper limits on orbital eccentricity of $0.07,0.04$ and 0.4 for WASP91b, WASP-105b and WASP-107b, respectively.

We present the system parameters from our final MCMC analyses in Table 2 and we plot the best fits to the radial-velocity data and the photometric data in Figs. 1-3.

\section{Discussion}

WASP-91b, a $1.34-M_{\text {Jup }}$ planet in a 2.8 -day orbit around a $\mathrm{K} 3 \mathrm{star}$, is the southern-most transiting planet known. WASP$105 \mathrm{~b}$ is a $1.8-M_{\text {Jup }}$ planet in a 7.9-day orbit around a K2 star. Finally, WASP-107b is a $0.12-M_{\text {Jup }}$ planet in a 5.7 -day orbit around a K6 star. Together with WASP-139b $\left(M_{\mathrm{P}}=0.12 \pm\right.$ $0.02 M_{\text {Jup }}$; Hellier et al. 2017), WASP-107b is the lowest-mass planet discovered by WASP to date; the next lowest are WASP29b (0.24 $M_{\text {Jup }}$; Hellier et al. 2010) and WASP-69b (0.26 $M_{\text {Jup }}$; Anderson et al. 2014). Giant planets seem to be more common around both stars of higher metallicity and stars of higher mass (e.g. Santos et al. 2004; Johnson et al. 2010). It is interesting to note that all three hosts are $\mathrm{K}$ stars and that WASP-91 and WASP-105 are metal rich, whilst the super-Neptune host, WASP-107, is solar metallicity.

WASP-91b (1.03 $\left.R_{\text {Jup }}\right)$ and WASP-105b $\left(0.96 R_{\text {Jup }}\right)$ are notable as having radii towards the lower end of the envelope for hot Jupiters (Fig. 6), though they are as expected from the empirical relation of Enoch et al. (2012) based on their semi-major axes and relatively low equilibrium temperatures. WASP-107b occupies a sparsely populated region in the planetary massradius diagram, with a mass 2.2 times that of Neptune and 0.40 times that of Saturn (Fig. 6). The planet's radius is toward the upper end of the super-Neptune/sub-Saturn regime and it is higher than expected from the empirical relation of Enoch et al. (2012) by around $0.30 R_{\mathrm{Jup}}$, perhaps suggestive of a low-metallicity composition.

\subsection{WASP-107b and the transition between ice giants and gas giants}

Under the core accretion model of planet formation, planetesimals coagulate to form a rocky core, which rapidly accretes a gaseous envelope once a critical mass of $\sim 10 M_{\text {Earth }}$ is reached 
Table 3. Periodogram analysis of the WASP lightcurves for WASP-107.

\begin{tabular}{llllrr}
\hline \hline Season & Dates & $N$ & $P[\mathrm{~d}]$ & $a$ [mmag] & \multicolumn{1}{c}{ FAP } \\
\hline 2009 & $4867-5010$ & 4029 & 1.134 & 0.003 & 0.056 \\
2010 & $5233-5376$ & 5315 & 17.17 & 0.004 & $<0.001$ \\
\hline
\end{tabular}

Notes. Observing dates are JD $-2450000, N$ is the number of observations used in the analysis, $a$ is the semi-amplitude of the best-fit sine wave at the period $P$ found in the periodogram with false-alarm probability FAP.
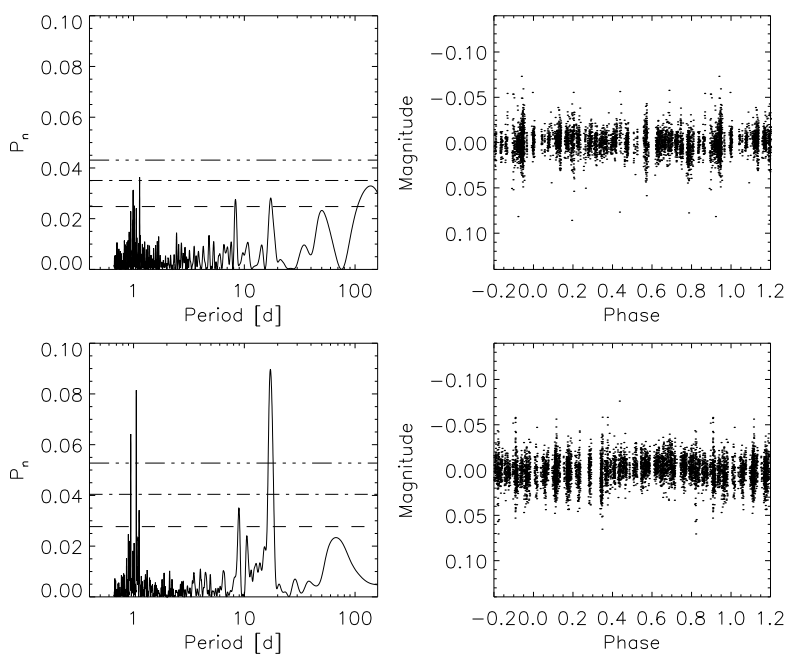

Fig. 5. Left: periodograms of the WASP lightcurves for WASP-107 obtained during 2009 (upper panel) and 2010 (lower panel). Horizontal lines indicate false-alarm probability levels $0.1,0.01$ and 0.001 . Right: lightcurves folded on the assumed rotation period of 17.1 days for data obtained during 2009 (upper panel) and 2010 (lower panel).

(Mizuno et al. 1978). One challenge for planet formation models is to explain why ice giants did not become gas giants.

Lambrechts et al. (2014) suggested core growth via the accretion of pebbles, rather than planetesimals, as a solution. Under this hypothesis, beyond a threshold mass, a core can halt the accretion of pebbles by gravitationally perturbing the surrounding disc. The gas envelope surrounding the core is then no longer supported by accretion heat and so rapidly collapses, resulting in a gas giant. Ice giants do not reach this threshold mass, which depends on orbital distance due to the steep increase in the gas scale height in flaring discs. This hypothesis offers a neat explanation for the bifurcation of the giants of the Solar System and it can be tested as it predicts both that ice giants in wide orbits are common relative to gas giants and that those gas giants are enriched (core mass $>50 M_{\oplus}$ ).

WASP-107b has a mass 2.2 times that of Neptune and 0.40 times that of Saturn, but a radius 0.94 times that of Jupiter. This suggests that WASP-107b is a low-mass gas giant, with a $\mathrm{H} / \mathrm{He}$-dominated composition (Fig. 6). We define a notional transition region between ice giants and gas giants which spans a planetary mass of between twice that of Neptune and half that of Saturn $\left(0.11 M_{\text {Jup }}<M_{\mathrm{P}}<0.15 M_{\text {Jup }}\right)$. We know of five planets ${ }^{1}$ with masses in that region: WASP-107b, WASP-139b (Hellier et al. 2017), HATS-7b (Bakos et al. 2015), HATS-8b

1 We excluded Kepler-9b and Kepler-9c as there is considerable uncertainty regarding their masses, which were inferred from transit-timing variations (Holman et al. 2010; Borsato et al. 2014; Hadden \& Lithwick 2014).

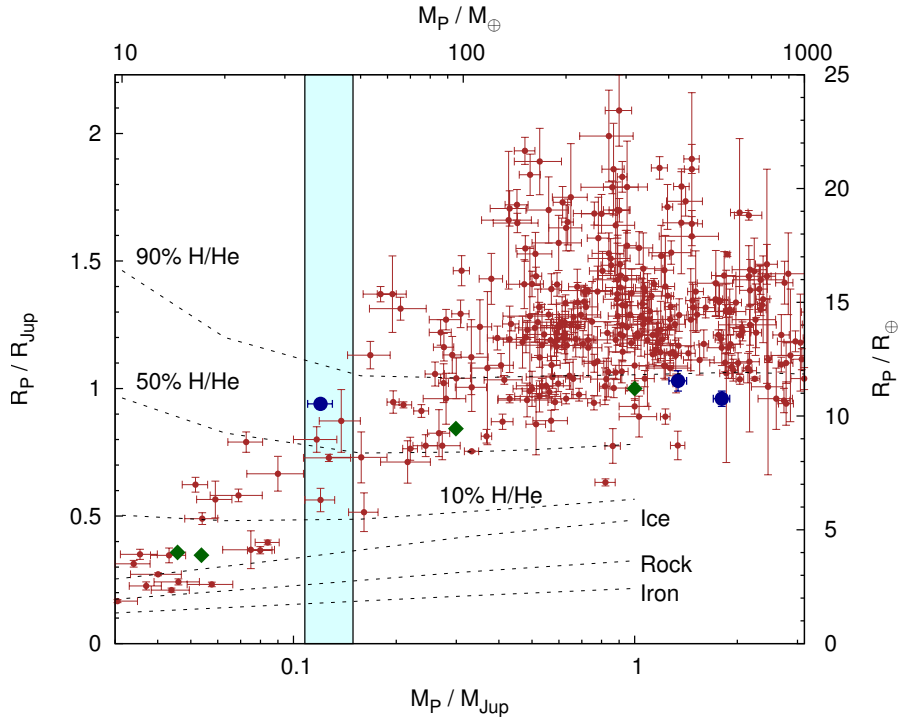

Fig. 6. Planetary mass-radius diagram showing the planets presented herein (blue circles), the Solar System giants (green diamonds) and the transiting exoplanets (red circles; data from TEPCat; masses measured by the radial-velocity technique to better than $20 \%$ precision). The dotted lines depict model planets of pure iron, rock and ice (from Fortney et al. 2007) and 3-Gyr isochrones for model planets with various $\mathrm{H} / \mathrm{He}$ fractions that are irradiated by a Sun-like star at $0.045 \mathrm{AU}$ (from Baraffe et al. 2008). The cyan rectangle indicates the notional transition region between ice giants and gas giants, where planetary mass is between twice that of Neptune and half that of Saturn.

(Bayliss et al. 2015), and the circumbinary planet Kepler-35b (Welsh et al. 2012). We may be able to discern whether these planets more closely resemble ice giants or gas giants by measuring their atmospheric metallicities, which is achievable by measuring their atmospheric $\mathrm{H}_{2} \mathrm{O}$ abundances via transmission spectroscopy with HST (e.g. Kreidberg et al. 2014). The atmospheric metallicities of Neptune and Uranus, the Solar System's ice giants, are far higher than that of Jupiter and Saturn, the Solar System's gas giants: with $\mathrm{C} / \mathrm{H} \approx 80$ times the proto-solar abundance as compared to 4-10 times (Guillot \& Gautier 2014). By measuring the atmospheric metallicity of planets in the transition region, we may gain insight into the planetesimal-accretion history of the planet and better understand both the formation pathways of ice giants and gas giants and the transition from one class to the other. For each planet in the transition region, we calculated their predicted atmospheric transmission signal (i.e. the product of the star's $K$-band flux and the area ratio of the planetary atmosphere's annulus to the stellar disc; Table 4). This suggests that WASP-107b is the most favourable target for transmission spectroscopy in the transmission region by an order of magnitude. Further, it is predicted to be an order of magnitude more favourable than WASP-43b, whose $\mathrm{H}_{2} \mathrm{O}$ abundance was measured recently (Kreidberg et al. 2014).

\subsection{The migration of short period, giant planets}

WASP-105b and WASP-107b could help us to understand the inward migration of hot Jupiters. To date, the orbits of planets in short orbits around cool stars $\left(T_{\text {eff }}<6250 \mathrm{~K}\right)$ have been found to be near-circular and near-aligned with the stellar spins, whereas planets in longer orbits, therefore experiencing weaker tidal forces, tend to be eccentric and/or misaligned (Albrecht et al. 2012; Anderson et al. 2015b). This has been 
Table 4. Planets in the transmission region between ice giants and gas giants.

\begin{tabular}{|c|c|c|c|c|c|c|c|c|c|}
\hline Planet & $\begin{array}{r}M_{\mathrm{P}} \\
\left(M_{\mathrm{Jup}}\right)\end{array}$ & $\begin{array}{r}R_{\mathrm{P}} \\
\left(R_{\mathrm{Jup}}\right)\end{array}$ & $\begin{array}{r}P \\
\text { (d) }\end{array}$ & $\begin{array}{r}M_{*} \\
\left(M_{\odot}\right)\end{array}$ & $\begin{array}{r}R_{*} \\
\left(R_{\odot}\right)\end{array}$ & $\begin{array}{l}T_{\text {eff }} \\
(\mathrm{K})\end{array}$ & $V$ & $K$ & $\begin{array}{c}\text { Transm } \\
\text { signal }\end{array}$ \\
\hline WASP-107b & $0.12 \pm 0.01$ & $0.94 \pm 0.02$ & 5.72 & $0.69 \pm 0.05$ & $0.66 \pm 0.02$ & $4430 \pm 120$ & 11.6 & 8.6 & 1000 \\
\hline WASP-139b & $0.12 \pm 0.02$ & $0.80 \pm 0.05$ & 5.92 & $0.92 \pm 0.10$ & $0.80 \pm 0.04$ & $5300 \pm 100$ & 12.4 & 10.5 & 94 \\
\hline HATS-7b & $0.12 \pm 0.01$ & $0.56 \pm 0.04$ & 3.19 & $0.85 \pm 0.03$ & $0.82 \pm 0.04$ & $4985 \pm 50$ & 13.3 & 11.0 & 21 \\
\hline HATS-8b & $0.14 \pm 0.02$ & $0.87_{-0.08}^{+0.12}$ & 3.58 & $1.06 \pm 0.04$ & $1.09_{-0.05}^{+0.15}$ & $5679 \pm 50$ & 14.0 & 12.7 & 10 \\
\hline Kepler-35b & $0.13 \pm 0.02$ & $0.73 \pm 0.01$ & 131.46 & $0.888 \pm 0.005$ & $1.028 \pm 0.002$ & $5606 \pm 150$ & 15.9 & 13.9 & 1 \\
\hline WASP-43b ${ }^{\dagger}$ & $2.03 \pm 0.05$ & $1.04 \pm 0.02$ & 0.81 & $0.72 \pm 0.03$ & $0.67 \pm 0.01$ & $4520 \pm 120$ & 12.4 & 9.3 & 74 \\
\hline
\end{tabular}

Notes. The data for WASP-107b are from this paper and the data for the other systems were taken from TEPCat. The transmission signal (final column) is the product of the star's $K$-band flux and the area ratio of the planetary atmosphere's annulus to the stellar disc; the transmission signal values were normalised such that the predicted signal for WASP-107b is $1000 .{ }^{(\dagger)}$ We included WASP-43b for comparison as its atmospheric water abundance was recently measured from a transmission spectrum (Kreidberg et al. 2014).

interpreted as evidence for high-eccentricity migration, in which a cold Jupiter is perturbed into an eccentric, misaligned orbit that is then circularized, shortened, and realigned by tidal dissipation (Albrecht et al. 2012). Whilst high-eccentricity migration may be responsible for a substantial fraction of hot Jupiters, there is growing evidence that some migrated inwards to their current orbits via interaction with a protoplanetary disc. For example, WASP-84b is in a relatively wide orbit around a young star, indicating that it has experienced relatively weak tidal forcing over a short duration. Therefore, its near-circular and near-aligned orbit is suggestive of disc migration (Anderson et al. 2014, 2015b). Further, WASP-47 comprises a hot Jupiter, a nearby super-Earth, a nearby Neptune, and a more distant Jupiter. The inner three planets are known to transit and the orbit of the hot Jupiter is near-aligned (Hellier et al. 2012; Sanchis-Ojeda et al. 2015; Becker et al. 2015; Neveu-VanMalle et al. 2016). It seems likely that disc migration operated in both of these hot-Jupiter systems.

By measuring the spin-orbit angle for a sample of planets in relatively wide orbits, for which tidal effects will be smaller, we can determine the relative contributions of migration pathways. With scaled orbital separations of $a / R_{*} \approx 18$, both WASP105 and WASP-107 lie beyond the empirical boundary between aligned and misaligned systems $\left(a / R_{*} \approx 15\right)$ and so would be interesting targets in that respect. If the planets underwent higheccentricity migration then we may expect their orbits to be eccentric and/or misaligned (e.g. Fabrycky \& Tremaine 2007), whereas near-circular and near-aligned orbits may be expected if they underwent planet-disc migration (e.g. Marzari \& Nelson 2009). We placed an upper limit of $e<0.04$ at $2 \sigma$ for WASP-105b, but the constraint is much weaker for WASP107b $(e<0.4$ at $2 \sigma$ ) due to the lower mass of the planet and therefore the smaller amplitude of the stellar reflex motion. With a scaled separation of $a / R_{*} \approx 9$, WASP-91b is expected to be in a near-aligned and near-circular orbit; indeed, we found $e<0.07$ at $2 \sigma$. The most common method employed to measure spin-orbit angles is to measure the apparent radialvelocity shift that occurs during transit (e.g. Albrecht et al. 2012). The predicted semi-amplitude of the RV shift is 20, 13 and $35 \mathrm{~m} \mathrm{~s}^{-1}$ for WASP-91, -105 and -107, respectively. An alternative possibility for WASP-107 is to infer the spin-orbit angle from the spot-crossing times measured over multiple transits (Sanchis-Ojeda et al. 2011; Nutzman et al. 2011). This could be done soon as WASP-107 was observed during Campaign 10 of the K2 mission (Howell et al. 2014; Kepler Guest Observer proposal 8060, PI: Anderson).
Acknowledgements. WASP-South is hosted by the South African Astronomical Observatory; we are grateful for their ongoing support and assistance. Funding for WASP comes from consortium universities and from the UK's Science and Technology Facilities Council. The Swiss Euler Telescope is operated by the University of Geneva, and is funded by the Swiss National Science Foundation. TRAPPIST is funded by the Belgian Fund for Scientific Research (Fond National de la Recherche Scientifique, FNRS) under the grant FRFC 2.5.594.09.F, with the participation of the Swiss National Science Fundation (SNF). M. Gillon and E. Jehin are FNRS Research Associates. L. Delrez acknowledges support from the Gruber Foundation Fellowship. This research has made use of the TEPCat catalogue of the physical properties of transiting planetary systems, which is maintained by John Southworth and is available at http://www . astro. keele. ac.uk/jkt/tepcat.

\section{References}

Albrecht, S., Winn, J. N., Johnson, J. A., et al. 2012, ApJ, 757, 18

Anderson, D. R., Collier Cameron, A., Gillon, M., et al. 2012, MNRAS, 422, 1988

Anderson, D. R., Collier Cameron, A., Delrez, L., et al. 2014, MNRAS, 445, 1114

Anderson, D. R., Collier Cameron, A., Hellier, C., et al. 2015a, A\&A, 575, A61 Anderson, D. R., Triaud, A. H. M. J., Turner, O. D., et al. 2015b, ApJ, 800, L9 Asplund, M., Grevesse, N., Sauval, A. J., \& Scott, P. 2009, ARA\&A, 47, 481 Bakos, G. Á., Penev, K., Bayliss, D., et al. 2015, ApJ, 813, 111

Baraffe, I., Chabrier, G., \& Barman, T. 2008, A\&A, 482, 315

Bayliss, D., Hartman, J. D., Bakos, G. Á., et al. 2015, AJ, 150, 49

Becker, J. C., Vanderburg, A., Adams, F. C., Rappaport, S. A., \& Schwengeler, H. M. 2015, ApJ, 812, L18

Borsato, L., Marzari, F., Nascimbeni, V., et al. 2014, A\&A, 571, A38

Claret, A. 2000, A\&A, 363, 1081

Claret, A. 2004, A\&A, 428, 1001

Collier Cameron, A., Pollacco, D., Street, R. A., et al. 2006, MNRAS, 373, 799

Collier Cameron, A., Wilson, D. M., West, R. G., et al. 2007, MNRAS, 380, 1230

Doyle, A. P., Smalley, B., Maxted, P. F. L., et al. 2013, MNRAS, 428, 3164

Enoch, B., Collier Cameron, A., \& Horne, K. 2012, A\&A, 540, A99

Fabrycky, D., \& Tremaine, S. 2007, ApJ, 669, 1298

Fortney, J. J., Marley, M. S., \& Barnes, J. W. 2007, ApJ, 659, 1661

Gillon, M., Doyle, A. P., Lendl, M., et al. 2011, A\&A, 533, A88

Gray, D. F. 2008, The Observation and Analysis of Stellar Photospheres (Cambridge University Press)

Guillot, T. 2005, Rev. Earth Planet. Sci., 33, 493

Guillot, T., \& Gautier, D. 2014, in Treatise on Geophysics, eds. T. Spohn, \& G. Schubert, ArXiv e-prints [arXiv: 1405.3752]

Hadden, S., \& Lithwick, Y. 2014, ApJ, 787, 80

Helled, R., \& Bodenheimer, P. 2014, ApJ, 789, 69

Hellier, C., Anderson, D. R., Collier Cameron, A., et al. 2010, ApJ, 723, L60

Hellier, C., Anderson, D. R., Collier Cameron, A., et al. 2012, MNRAS, 426, 739

Hellier, C., Anderson, D. R., Collier Cameron, A., et al. 2017, 2017, MNRAS, 465,3693

Holman, M. J., Fabrycky, D. C., Ragozzine, D., et al. 2010, Science, 330, 51 
Howell, S. B., Sobeck, C., Haas, M., et al. 2014, PASP, 126, 398

Jehin, E., Gillon, M., Queloz, D., et al. 2011, The Messenger, 145, 2

Johnson, J. A., Aller, K. M., Howard, A. W., \& Crepp, J. R. 2010, PASP, 122, 905

Kreidberg, L., Bean, J. L., Désert, J.-M., et al. 2014, ApJ, 793, L27

Lambrechts, M., Johansen, A., \& Morbidelli, A. 2014, A\&A, 572, A35

Lendl, M., Anderson, D. R., Collier-Cameron, A., et al. 2012, A\&A, 544, A72

Lin, D. N. C., Bodenheimer, P., \& Richardson, D. C. 1996, Nature, 380, 606

Mandel, K., \& Agol, E. 2002, ApJ, 580, L171

Marzari, F., \& Nelson, A. F. 2009, ApJ, 705, 1575

Maxted, P. F. L., Anderson, D. R., Collier Cameron, A., et al. 2011, PASP, 123 , 547

Maxted, P. F. L., Serenelli, A. M., \& Southworth, J. 2015, A\&A, 575, A36
Mizuno, H., Nakazawa, K., \& Hayashi, C. 1978, Prog. Th. Phys., 60, 699 Neveu-VanMalle, M., Queloz, D., Anderson, D. R., et al. 2016, A\&A, 586, A93 Nutzman, P. A., Fabrycky, D. C., \& Fortney, J. J. 2011, ApJ, 740, L10 Pollacco, D. L., Skillen, I., Cameron, A. C., et al. 2006, PASP, 118, 1407 Pollack, J. B., Hubickyj, O., Bodenheimer, P., et al. 1996, Icarus, 124, 62 Queloz, D., Mayor, M., Weber, L., et al. 2000, A\&A, 354, 99 Rasio, F. A., \& Ford, E. B. 1996, Science, 274, 954

Sanchis-Ojeda, R., Winn, J. N., Holman, M. J., et al. 2011, ApJ, 733, 127 Sanchis-Ojeda, R., Winn, J. N., Dai, F., et al. 2015, ApJ, 812, L11 Santos, N. C., Israelian, G., \& Mayor, M. 2004, A\&A, 415, 1153

Welsh, W. F., Orosz, J. A., Carter, J. A., et al. 2012, Nature, 481, 475 Winn, J. N., Fabrycky, D., Albrecht, S., \& Johnson, J. A. 2010, ApJ, 718 , L145 